J. Clin. Chem. Clin. Biochem.

Vol. 15, 1977, pp. 311-318

\title{
Geschlechtsspezifische Wirkung von Insulin auf den Glucosemetabolismus lymphatischer Gewebe von Ratte und Mensch
}

\author{
Von H. Bojar, Marieluise Unnewehr, K. Balzer und W. Staib \\ Institut für Physiologische Chemie II der Universität Düsseldorf
}

(Eingegangen am 17. Juli 1976/11. Januar 1977)

Herrn Prof. Dr. L. Birkofer zum 65. Geburtstag gewidmet

Zusammenfassung: Insulin kontrolliert den Glucosestoffwechsel lymphatischer Zellen aus Thymus und Milz der Ratte. Für die Wirkung des Hormons auf die Glucoseaufnahme isolierter Zellen aus beiden lymphatischen Organen konnte eine signifikante Geschlechtsspezifität nachgewiesen werden. Insulin stimulierte in Konzentrationen von 0,1 bis $10 \mathrm{nmol} / \mathrm{l}$ die Glucoseaufnahme der Zellen männlicher Tiere, während es bei Zellen weiblicher Ratten den Glucoseeinstrom hemmte. Die Lactatbildung wurde dagegen in lymphatischen Zellen männlicher und weiblicher Tiere gesteigert. Cortisol ( $100 \mathrm{nmol} / \mathrm{l})$ entfaltete eine Antiinsulin-Wirkung auf Glucoseaufnahme und Lactatfreisetzung isolierter Thymocyten männlicher Ratten. Bei Zellen weiblicher Tiere hemmten Insulin und Cortisol den Glucoseeinstrom, während sie auf die Lactatbildung antagonistisch wirkten. Der geschlechtsspezifische stimulatorische Einfluß des Insulins auf die Gluocseaufnahme der Thymocyten männlicher Ratten konnte durch Kastration in eine für weibliche Tiere charakteristische hormoninduzierte Hemmung des Glucoseeinstroms umgewandelt werden. Eine Altersabhängigkeit der Geschlechtsspezifität der Insulinwirkung auf die Glucoseaufnahme wurde nachgewiesen. An Thymocyten männlicher und weiblicher Ratten mit einem Körpergewicht von $10 \mathrm{~g}$ und $40 \mathrm{~g}$ konnte eine Geschlechtsabhängigkeit der Insulinwirkung gezeigt werden, die der bei 140-150 g schweren Tieren entsprach. Bei $80 \mathrm{~g}$ schweren Ratten wurde dagegen die Glucoseaufnahme der Thymocyten männlicher und weiblicher Ratten stimuliert.

Vergleichbare geschlechtsspezifische Differenzen in der insulinabhängigen Regulation des Glucosestoffwechsels wurden auch an isolierten Zellen des präpubertalen menschlichen Thymus nachgewiesen. Thymocyten von Jungen reagierten auf Insulin mit gesteigerter Glucoseaufnahme und Lactatbildung, während die Zellen von Mädchen mit einer Hemmung der Glucoseaufnahme verbunden mit einer Stimulation der Lactatbildung reagierten. Die Altersabhängigkeit und Geschlechtsspezifität der Insulinwirkung auf den Glucosemetabolismus lymphatischer Zellen könnten ein Grund für die widersprechenden Resultate anderer Autoren sein.

\section{Sex specific action of insulin on the glucose metabolism of lymphatic tissue of rats and humans}

Summary: Glucose metabolism of lymphoid cells isolated from thymus and spleen of Wistar rats, weighing $140-150 \mathrm{~g}$, was found to be sensitive to insulin. The influence of insulin on glucose uptake by isolated cells from both lymphoid tissues displayed a significant sex specificity. Insulin at $0.1-10 \mathrm{nmol} / 1$ stimulated glucose uptake of cells from male rats, whereas in cells from females, inhibition of glucose uptake was observed. However, lactate production was enhanced in lymphoid cells of both sexes. Cortisol $(100 \mathrm{nmol} / \mathrm{l})$ displayed a significant anti-insulin action on glucose uptake and lactate release by thymocytes of male rats. In contrast, in cells from females, cortisol and insulin both exhibited an inhibitory action on glucose uptake, whereas the hormones were found to antagonise lactate production. The sex specific stimulatory influence of insulin on glucose uptake by thymocytes of male rats was reversed and the hormone became inhibitory when animals were castrated. The action of insulin on glucose uptake was also shown to be agedependent. In experiments with raț weighing either about $10 \mathrm{~g}$ or $40 \mathrm{~g}$, sex-specific effects on glucose influx were found, that were similar to those of rats weighing $140-150 \mathrm{~g}$. However, stimulation of glucose uptake was found with thymocytes from rats of both sexes weighing about $80 \mathrm{~g}$.

Comparable results were obtained with isolated thymocytes from immature humans ( 2 months -10 years old). Incubation of thymocytes from males with insulin $(10 \mathrm{nmol} / \mathrm{l})$ stimulated glucose uptake and lactate release, whereas insulin caused an inhibition of glucose uptake and an enhancement of lactate production in thymocytes from females. Age dependence and sex specificity of insulin action on glucose metabolism in lymphoid cells and tissues may explain the contradictory results reported by other authors. 
Einleitung

Zum Problem der hormonellen Kontrolle lymphatischer Zellen und Gewebe durch Insulin sind eine Reihe sich widersprechender Untersuchungen veröffentlicht worden. An der Oberfläche zirkulierender Lymphocyten des Menschen konnten Gavin et al. $(1,2)$ spezifische Insulinrezeptoren mit hoher Affinität nachweisen, wie sie von Leberplasmamembranen $(3,4)$ und Fettzellen $(4,5)$ bekannt sind. Die physiologische Bedeutung dieser spezifischen Hormonbindung blieb unklar, weil Untersuchungen zur Wirkung von Insulin auf den Stoffwechsel gereinigter peripherer Lymphocyten und nicht fraktionierter Leukocytenpräparationen zu uneinheitlichen und teilweise widersprechenden Ergebnissen führten (6, 7, 8, 9, 10). So konnte zum Beispiel nicht eindeutig geklärt werden, ob eine diabetische Stoffwechsellage Voraussetzung für einen Insulineffekt auf die Glucoseverwertung ist. Auch die Existenz der Insulinrezeptoren auf der Plasmamembran normaler peripherer Lymphocyten ist in Frage gestellt worden. Krug et al. (11) fanden keine spezifische Bindung von Insulin an frisch isolierte Lymphocyten aus dem menschlichen Blut, beschrieben aber das Erscheinen von Insulinrezeptoren nach in vitro-Transformation der peripheren Lymphocyten mit Concanavalin A und Cytochalasin B. Untersuchungen der Insulinsensibilität verschiedener lymphatischer Gewebe führten ebenfalls zu unterschiedlichen Resultaten. Während Helmreich et al. (12) keinen Effekt auf die Glucoseutilisation von Lymphocyten aus Meerschweinchenlymphknoten fanden, konnten Boyett et al. (13) einen Insulineinfluß auf den Glucosestoffwechsel isolierter Thymocyten aus adrenalektomierten Ratten nachweisen. Goldfine et al. (14) beschrieben eine Stimulation des $\alpha$-Aminoisobuttersäuretransportes und spezifische Insulinbindungsstellen an Thymocyten säugender Ratten. Die Widersprüchlichkeit der Untersuchungen zur Insulinsensibilität lymphatischer Zellen und Gewebe und die auffallende Heterogenität des Untersuchungsmaterials (fertile adrenalektomierte Ratten (13), säugende Ratten (14), diabetische Probanden (7)), bei dem eine Insulinempfindlichkeit nachgewiesen werden konnte, lassen vermuten, daß die Insulinsensibilität lymphatischer Zellen einer starken Modulation durch verschiedene endogene Faktoren unterliegt. Gegenstand der vorliegenden Untersuchungen ist einerseits eine Analyse der Insulinempfindlichkeit isolierter Thymocyten intakter, nicht diabetischer Ratten in Abhängigkeit von Geschlecht und Alter der Tiere. Andererseits versuchten wir zu klären, ob der Rattenthymus in Hinblick auf die Insulinsensibilität eine Ausnahme darstellt oder ob auch andere lymphatische Organe der Ratte wie z. B. die Milz als Zielorgane für Insulin anzusehen sind. Anschließend überprüften wir an isolierten Thymocyten nicht diabetischer Kinder die Wirksamkeit von Insulin auf den Glucosestoffwechsel des präpubertalen menschlichen Thymus.

\section{Material und Methoden}

\section{Untersuchungsmaterial}

Zur Isolierung tierischer Thymus- und Milzzellen wurden nichtdiabetische, mit Standardkost (Firma Höveler/Langenfeld) ernährte Wistar-II-Ratten $(10-150 \mathrm{~g})$ beiderlei Geschlechts verwendet. Menschliche Thymocyten wurden aus frisch entnommenen Gewebsproben nicht diabetischer Kinder im Alter von 2 Monaten bis zu 10 Jahren isoliert. Die Entnahme der Proben erfolgte bei Herzoperationen in der kardiochirurgischen Abteilung der Universitätskliniken Düsseldorf.

\section{Reagenzien}

Wenn nicht anders angegeben, wurden Chemikalien der Firma Merck, Darmstadt, vom Reinheitsgrad p. a. verwendet. Hydrocortison wurde von der Firma Calbio chem, San Diego, Kalifornien, USA, bezogen. Kristallines Rinderinsulin wurde uns freundlicherweise von Herrn Dr. Brandenburg, Deutsches Wollforschüngsinstitut, Aachen, zur Verfügung gestellt.

\section{Isolierungsmethode}

Die Tiere wurden mit Hexobarbital (Evipan, Bayer-Leverkusen, $0,02 \mathrm{~g} / 100 \mathrm{~g}$ Körpergewicht, intraperitoneal) narkotisiert und erhielten gleichzeitig Liquemin (Hoffmann-La Roche AG, Grenzach, 500 USP:E Heparin/100 g Körpergewicht, intraperitoneal). Thymi von jeweils 5-10 Ratten wurden in einer eisgekühlten Petrischale von anhaftendem Bindegewebe freipräpariert. Anschließend wurden die Organe mehrfach incidiert. Nach Zugabe von $10 \mathrm{ml}$ calciumfreiem Krebs-RingerPhosphat-Puffer (pH 7,4) wurden die Zellen durch vorsichtiges Ausstreichen mit einem Siliconschlauch suspendiert.

Die Zellsuspension wurde durch ein Nylongewebe von 150 $\mu \mathrm{m}$ Maschenweite filtriert und auf ein Volumen von $20 \mathrm{ml}$ aufgefüllt. Anschließend wurden die Zellen abzentrifugiert (3000 U/min, $5 \mathrm{~min}, 4^{\circ} \mathrm{C}$, Sorvall RC2-B, Rotor SS34). Der Waschvorgang wurde zweimal wiederholt. Das Sediment wurde zur Bestimmung des Feuchtgewichtes gewogen und in dem neunfachen Volumen (Gewicht/Volumen) Krebs-RingerBicarbonat-Puffer (pH 7,4) aufgenommen.

Die Milz wurde nach Kanülierung des Truncus coeliacus und Unterbindung der Arteria hepatica und Arteria gastrica sinistra mit $40 \mathrm{ml}$ calciumfreien Krebs-Ringer-PhosphatPuffer (pH 7,4) in situ perfundiert. Anschließend wurden Milzzellensuspensionen nach dem zur Thymocytenpräparation angewendeten Verfahren hergestellt.

Menschliche Thymocytensuspensionen wurden nach der gleichen Methode gewonnen.

\section{Versuchsdurch führung}

Jeweils $0,4 \mathrm{ml}$ der Zellsuspension wurde in ein $20 \mathrm{ml}$ Polyethylenröhrchen (Packard Instrument GmbH, Frankfurt) zu 0,5 ml Inkubationsmedium pipettiert, das die angegebenen Substrate und Hormone in einem Krebs-Ringer--Bicarbonat-Puffer $(\mathrm{pH} \mathrm{7,4)} \mathrm{ent-}$ hielt. Die Röhrehen wurden mit Carbogen (95 Vol. \% $\mathrm{O}_{2}, 5$ Vol. $\% \mathrm{CO}_{2}$ ) begast, verschraubt und in einem Schüttelbad (Frequenz $110-120 / \mathrm{min}$ ) bei $37^{\circ} \mathrm{C}$ inkubiert. Die Reaktion wurde durch $0,1 \mathrm{ml}$ einer $300 \mathrm{~g} / 1$ Perchlorsäure gestoppt. Nach zweiminütiger Zentrifugation (Eppendorf Tischzentrifuge, Modell 3200) wurde der Überstand mit $\mathrm{KHCO}_{3}$ in Substanz neutralisiert. Nach erneuter Zentrifugation wurden Glucose (15) und Lactat (16) im Überstand bestimmt.

\section{Statistik}

Die statistischen Auswertungen erfolgten nach dem t-Tët nach Student.

\section{Ërgebnisse}

In Abbildung 1 ist die Glucoseaufnahme und Lactatbildung isolierter Thymocyten $140-150 \mathrm{~g}$ schwerer Ratten beiderlei Geschlechts in Abhängigkeit von der 

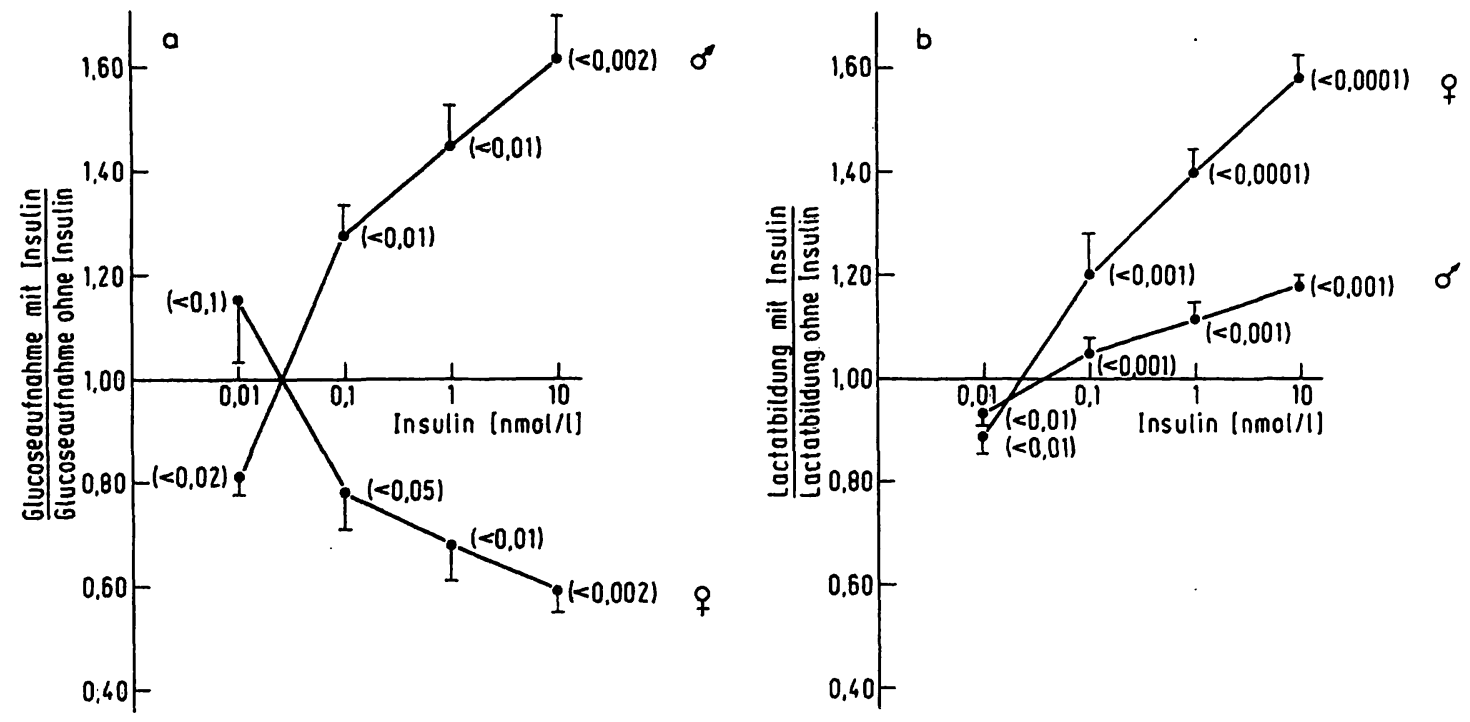

Abb. 1. Insulinwirkung auf die Glucoseaufnahme (a) und Lactatbildung (b) isolierter Thymocyten männlicher und weiblicher Ratten (Gewicht: $140-150 \mathrm{~g}$ ).

$40 \mathrm{mg}$ Thymocyten wurden in $0,9 \mathrm{ml}$ Krebs-Ringer-Bicarbonatpuffer mit 5,5 mmol/l Glucose und steigenden Insulinkonzentrationen 2 Stunden bei $37^{\circ} \mathrm{C}$ inkubiert.

a) Die Glucoseaufnahme in den Kontrollansätzen (ohne Insulin) betrug bei Thymocyten männlicher Tiere 14,61 $\pm 1,08 \mu$ mol/ $2 \mathrm{~h} \cdot \mathrm{g}$ Feuchtgewicht und bei Thymocyten weiblicher Tiere 12,73 $\pm 1,24 \mu \mathrm{mol} / 2 \mathrm{~h} \cdot \mathrm{g}$ Feuchtgewicht.

b) Die Lactatbildung in den Kontrollen (ohne Insulin) betrug bei den Zellen männlicher Tiere 6,08 $\pm 0,55 \mu \mathrm{mol} / 2 \mathrm{~h} \cdot \mathrm{g}$ Feuchtgewicht und bei den Zellen weiblicher Tiere 3,56 $\pm 0,20 \mu \mathrm{mol} / 2 \mathrm{~h} \cdot \mathrm{g}$ Feuchtgewicht.

Die Werte sind Mittelwerte $\left(\overline{\mathbf{x}} \pm s_{\overline{\mathbf{x}}}\right)$ aus 5 Präparationen. Die p-Werte für die Differenzen zwischen den Ansätzen mit und ohne Insulin (Kontrollen) sind in Klammern angegeben.

Tab. 1. Insulinwirkung auf die Glucoseaufnahme und Lactatbildung isolierter Thymocyten männlicher, weiblicher und kastrierter männlicher Ratten.

$40 \mathrm{mg}$ Zellen wurden in $0,9 \mathrm{ml}$ Krebs-Ringer-Bicarbonatpuffer mit $1,1 \mathrm{mmol} / 1$ Glucose und $10 \mathrm{nmol} / 1 \mathrm{Insulin} 60 \mathrm{~min}$ bei $37^{\circ} \mathrm{C}$ inkubiert.

Die Werte sind Mittelwerte $\left(\overline{\mathbf{x}} \pm 5_{\bar{x}}\right)$ aus 5-8 Präparationen. Die p-Werte für die Quotienten zwischen der Glucoseaufnahme in den Ansätzen mit und ohne Insulin (Kontrollen) sind in Klammern angegeben.

\begin{tabular}{|c|c|c|c|c|}
\hline & \multicolumn{2}{|c|}{ Glucoseaufnahme } & \multicolumn{2}{|c|}{ Lactatbildung } \\
\hline & Kontrolle & Insulin & Kontrolle & Insulin \\
\hline & \multicolumn{2}{|c|}{$\mu \mathrm{mol} / \mathrm{h} \cdot \mathrm{g}$ Feuchtgewicht } & \multicolumn{2}{|c|}{$\mu \mathrm{mol} / \mathrm{h} \cdot \mathrm{g}$ Feuchtgewicht } \\
\hline \multirow[t]{2}{*}{ männlich } & $0,90 \pm 0,15$ & $1,32 \pm 0,24$ & $1,20 \pm 0,18$ & $1,70 \pm 0,22$ \\
\hline & $1,467(<0,0$ & & $1,417(<0,0$ & \\
\hline \multirow[t]{2}{*}{ weiblich } & $2,55 \pm 0,20$ & $1,50 \pm 0,17$ & $1,07 \pm 0,06$ & $2,07 \pm 0,15$ \\
\hline & $-0,588<<$ & & $1,935(<0,0$ & \\
\hline \multirow{2}{*}{$\begin{array}{l}\text { männlich } \\
3 \text { Tage nach } \\
\text { Kastration }\end{array}$} & $1,79 \pm 0,30$ & $1,21 \pm 0,75$ & $1,04 \pm 0,07$ & $1,39 \pm 0,09$ \\
\hline & \multicolumn{2}{|c|}{$-0,676(<0,0001)$} & \multicolumn{2}{|c|}{$1,337(<0,0001)$} \\
\hline
\end{tabular}

Insulinkonzentration im İnkubationsmedium dargestellt. Bei Thymocyten männlicher Ratten bewirkte 10 pmol/1 Insulin eine Hemmung der Glucoseaufnahme, während das Hormon in einem Konzentrationsbereich von 0,1 bis $10 \mathrm{nmol} / 1$ zu einer signifikanten Erhöhung des Glucoseeinstroms führte (Abb. 1 a). In eindrucksvollem Gegensatz zu Thymocyten männlicher Ratten reagierten Zellen weiblicher Tiere auf $0,1-10 \mathrm{nmol} / 1 \mathrm{Insulin} \mathrm{mit}$ einer deutlichen Hemmung der Glucoseaufnahme. Eine angedeutete Stimulation der Glucoseutilisation in Gegenwart von $10 \mathrm{pmol} / 1$ Insulin ließ sich dagegen nicht statistisch sichern. Der Vergleich der Dosis-Wirkungs-Kurven männlicher und weiblicher Ratten (Abb. 1 a) demonstriert die in einem weiten Konzentrationsbereich hochsignifikante Geschlechtsspezifität des Insulineffektes auf den Glucoseeinstrom. Abbildung $1 \mathrm{~b}$ zeigt den Einfluß von 
Isulin auf die Lactatbildung. Bei einer Insulinkonzentration von $10 \mathrm{pmol} / \mathrm{l}$ war die Lactatfreisetzung bei Thymocyten männlicher und weiblicher Tiere geringgradig vermindert. In Konzentrationen von $0,1-10 \mathrm{nmol} / \mathrm{l}$ bewirkte Insulin dagegen eine signifikante Steigerung der Lactatbildung der isolierten Zellen des männlichen und weiblichen Tierkollektivs. Während bei Thymocyten männlicher Ratten durch Insulin $(0,1-10 \mathrm{nmol} / \mathrm{l})$ Glucoseaufnahme und Lactatfreisetzung gleichsinnig beeinflußt werden, reagierten Thymocyten weiblicher Tiere auf Insulin trotz einer partiell gehemmten Glucoseaufnahme mit einer eindeutigen Erhöhung der Lactatbildung.

Um zu überprüfen, ob die an Thymocyten beobachtete Geschlechtsspezifität des Insulineffektes auf die Glucoseaufnahme auch an anderen Zellen des lymphatischen Systems nachweisbar ist, wurden vergleichende Untersuchungen an isolierten Milzzellen männlicher und weiblicher Ratten durchgeführt. Abbildung 2 zeigt Dosis-Wirkungs-Kurven für die Effekte von Insulin auf den Glucoseverbrauch und die Lactatfreisetzung isolierter Milzzellen bei verschiedenen Glucosekonzentrationen im Inkubationsmedium. In Gegenwart von
5,5 mmol/1 Glucose (Abb. 2a, b) konnte, wie bei Thymocytensuspensionen, die Wirkung von Insulin auf die Glucoseaufnahme und Lactatbildung nach zweistündiger Inkubation der Zellen statistisch gesichert werden. Nach Erniedrigung der Glucosekonzentration des Mediums auf $1,1 \mathrm{mmol} / 1$ (Abb. 2c, d) war es bereits nach einer Stunde technisch möglich, dosisabhängige signifikante Veränderungen des Glucoseeinstroms unter Insulin zu ermitteln, die von signifikanten Effekten auf die Lactatfreisetzung begleitet wurden. Ein Vergleich der Abbildung 2 mit Abbildung 1 läßt die weitgehende Analogie der an isolierten Milz- und Thymuszellen ermittelten Dosis-Wirkungs-Kurven erkennen. Die an Thymocyten beobachtete überraschende geschlechtsspezifische Regulation der Glucoseaufnahme durch Insulin läßt sich ebenso eindeutig auch an isolierten Milzzellen der Ratte nachweisen. Im Gegensatz zu den Experimenten an isolierten Thymocyten (Abb. 1), konnte an Milzzellen weiblicher Tiere der hemmende Insulineffekt auci bei einer Hormonkonzentration von nur $10 \mathrm{pmol} / 1$ statistisch gesichert werden. In Analogie zu den Thymocytenversuchen bewirkte $10 \mathrm{pmol} / \mathrm{l}$ Insulin bei männlichen Milzzellen eine Hemmung der Glucoseaufnahme, die sich allerdings nicht statistisch
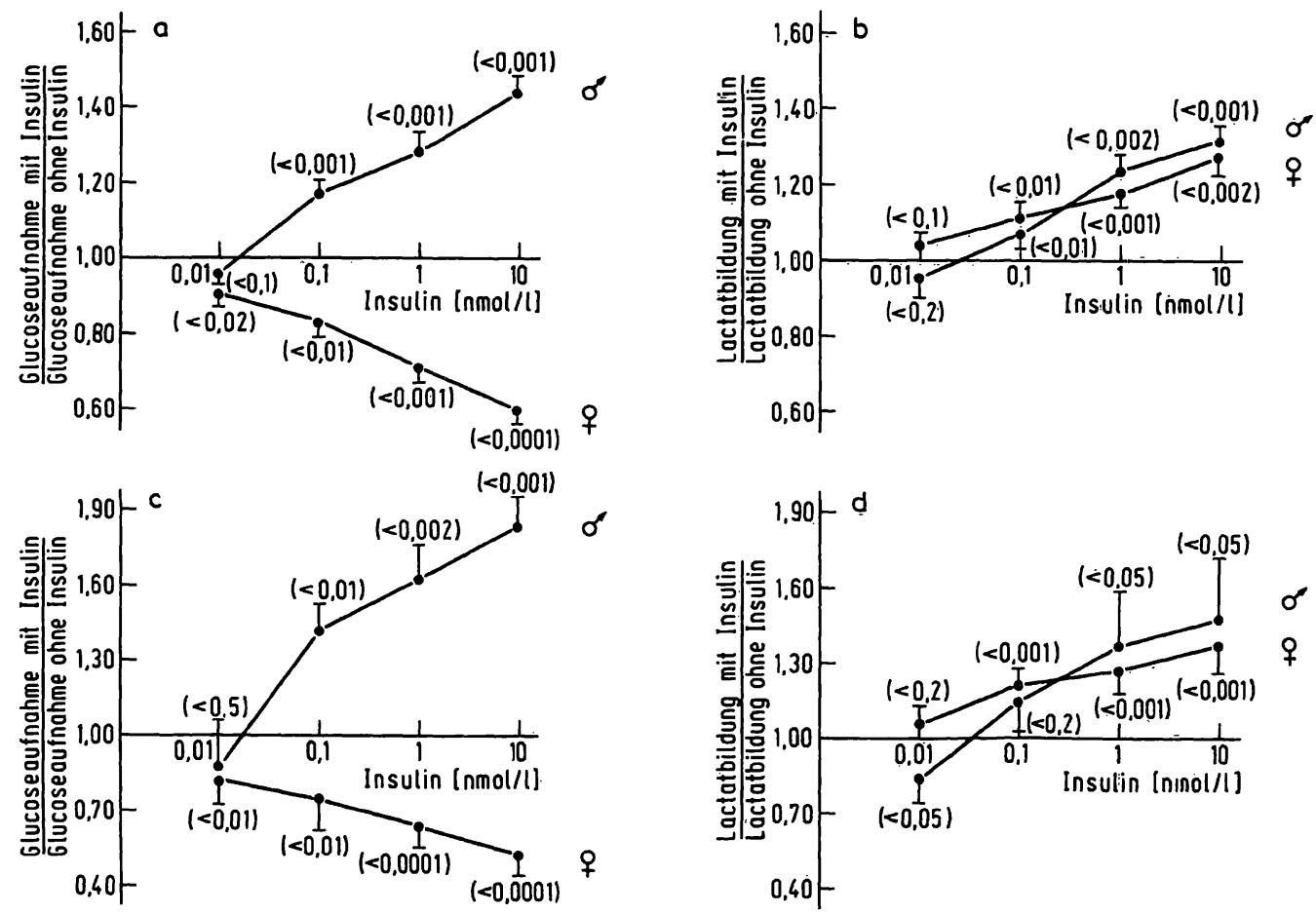

Abb. 2. Insulinwirkung auf die Glucoseaufnahme und Lactatbildung isolierter Milzzellen männlicher und weiblicher Ratten (Gewicht: $140-150 \mathrm{~g}$ ) bei verschiedenen Glucosekonzentrationen im Medium. $40 \mathrm{mg}$ Zellen wurden in $0,9 \mathrm{ml}$ Krebs-Ringer-BicarbonatPuffer in Gegen wart steigender Insulinkonzentrationen bzw. ohne Insulin (Kontrollansätze) inkubiert.

(a) Glucoseaufnahme und (b) Lactatbildung in Anwesenheit von 5,5 mmol/1 Glucose; Inkubationsbedingungen: $2 \mathrm{~h}, 37^{\circ} \mathrm{C}$. (c) Glucoseaufnahme und (d) Lactatbildung in Gegenwart von $1,1 \mathrm{mmol} / 1$ Glcuose; Inkubationsbedingungen: $1 \mathrm{~h}, 37^{\circ} \mathrm{C}$. In den Kontrollansätzen betrug die Glucoseaufnahme bzw. Lactatbildung bezogen auf Gramm Feuchtgewicht

(a) o 14,69 $\pm 1,07 \mu \mathrm{mol}$ Glucose $/ 2 \mathrm{~h}, \$ 18,29 \pm 2,10 \mu \mathrm{mol}$ Glucose $/ 2 \mathrm{~h}$,

(b) o $5,20 \pm 0,31 \mu \mathrm{mol} \mathrm{Lactat} / 2 \mathrm{~h}, \$ 5,82 \pm 0,37 \mu \mathrm{mol} \mathrm{Lactąt} / 2 \mathrm{~h}$,

(c) $\delta 1,98 \pm 0,28 \mu \mathrm{mol}$ Glucose $/ \mathrm{h}, ~ \% 2,49 \pm 0,21 \mu \mathrm{mol} \mathrm{Glucose} / \mathrm{h}$

(d) o $4,60 \pm 0,82 \mu \mathrm{mol} \mathrm{Lactat} / \mathrm{h}, \$ 2,10 \pm 0,1 \mu \mathrm{mol} \mathrm{Lactat} / \mathrm{h}$.

Die Werte sind Mittelwerte $(\bar{x} \pm s \bar{x})$ aus 5-9 Präparationen. Die p-Werte für die Differenzen zwischẹn den Ansätzen mit und ohne Insulin (Kontrollansätze) sind in Klammern angegeben. 
absichern ließ. Die Lactatbildung wird in Zellen beider lymphatischer Organe bei männlichen und weiblichen Tieren durch 0,1-10 nmol/1 Insulin stimuliert (Abb. $1,2)$. Wälurend Thymocyten männlicher und weiblicher Ratten auf 10 pmol/1 Insulin mit einer geringfügigen, aber signifikanten Hemmung der Lactatfreisetzung reagierten, war die Insulin reaktivität der Lactatbildung durch Milzzellen von Tieren beiderlei Geschlechts bis auf eine Ausnahme statistisch nicht zu sichern (Abb. 2). Die Lactatbildung wird dagegegen in Zellen beider lymphatischer Organe bei männlichen und weiblichen Tieren durch Insulin stimuliert. In Abbildung 3 ist der Einfluß von Insulin und Cortisol auf den Glucosestoffwechsel isolierter Thymocyten männlicher (nicht schraffierte Säulen) und weiblicher (schraffierte Säulen) Ratten dargestellt. Die von Matsui et al. (17) für Mausohrstreifen-Präparate beschriebene Antiinsulinwirkung des Cortisols konnte auch für Thymocyten männlicher Ratten nachgewiesen werden. Während $1 \mathrm{nmol} / 1$ Insulin die Glucoseaufnahme (Abb. 3a) und Lactatproduktion (Abb. 3b) männlicher Thymocy ten steigerte, hemmte Cortisol in einer Konzentration von $100 \mathrm{nmol} / \mathrm{l}$ Glucoseeinstrom und Lactatfreise tzung. Bei gleichzeitiger Anwesenheit beider Hormone im Inkubationsmedium konnte Cortisol die Wirkung des Insulins zwar abschwächen, aber trotz einhundertfach höherer Konzentration nicht aufikeben. Cortisol wirkte dagegen nicht insulinantagonistisch auf die Glucoseaufnahme der Thymocyten weiblicher Tiere (Abb. 3a). Insulin ( $1 \mathrm{nmol} / \mathrm{l})$ hemmte die Glucoseaufnahme um - $26 \%$ der Kontrollen, Cortisol $(100 \mathrm{nmol} / \mathrm{l})$ um $-44 \%$. Enthielt das Inkubationsmedium beide Hormone in den angegebenen Konzentrationen, wurde die Glucoseaufnahme im Vergleich zu den Kontrollen ohne Hormon um - $34 \%$ gehemmt. Dagegen konnte bei weiblichen wie bei männlichen Tieren eine signifikante Antiinsulinwirkung des Cortisols auf die Lactatbildung nachgewiesen werden.

Der Einfluß einer Kastration auf die geschlech tsspezifische Steuerung der Glucoseaufnahme durch Insulin ist in der Tabelle dargestellt. Thymocyten kastrierter männlicher Ratten reagierten auf $10 \mathrm{nmol} / 1$ Insulin in Analogie zu den Zellen weiblicher Tiere mit einer hochsignifikanten Drosselung des Glucoseeinstroms. Trotz der verminderten Glucoseaufnahme war die Lactatfreisetzung erhöht. Der Insulineffekt auf die Lactatbildung entsprach bei den kastrierten männlichen Ratten $(+33,7 \%)$ etwa dem der männlichen Kontrollgruppe $(+41,7 \%)$ und war damit erheblich schwächer als bei den Zellen des weiblichen Vergleichskollektivs (+ 93,5\%).

Im Verlauf unserer Untersuchungen fanden wir Anzeichen für eine mögliche Altersabhängigkeit der geschlechtsspezifischen Regulation der Glucoseaufnahme unter Insulin. Abbildung 4 zeigt den Einfluß von $10 \mathrm{nmol} / 1$ Insulin auf den Glucoseeinstrom in isolierte Thymocyten männlicher und weiblicher Ratten in Abhängigkeit vom Körpergewicht der Tiere. Thymocyten 10, 20, 80 und $150 \mathrm{~g}$ schwerer männlicher Ratten reagierten auf Insulin mit
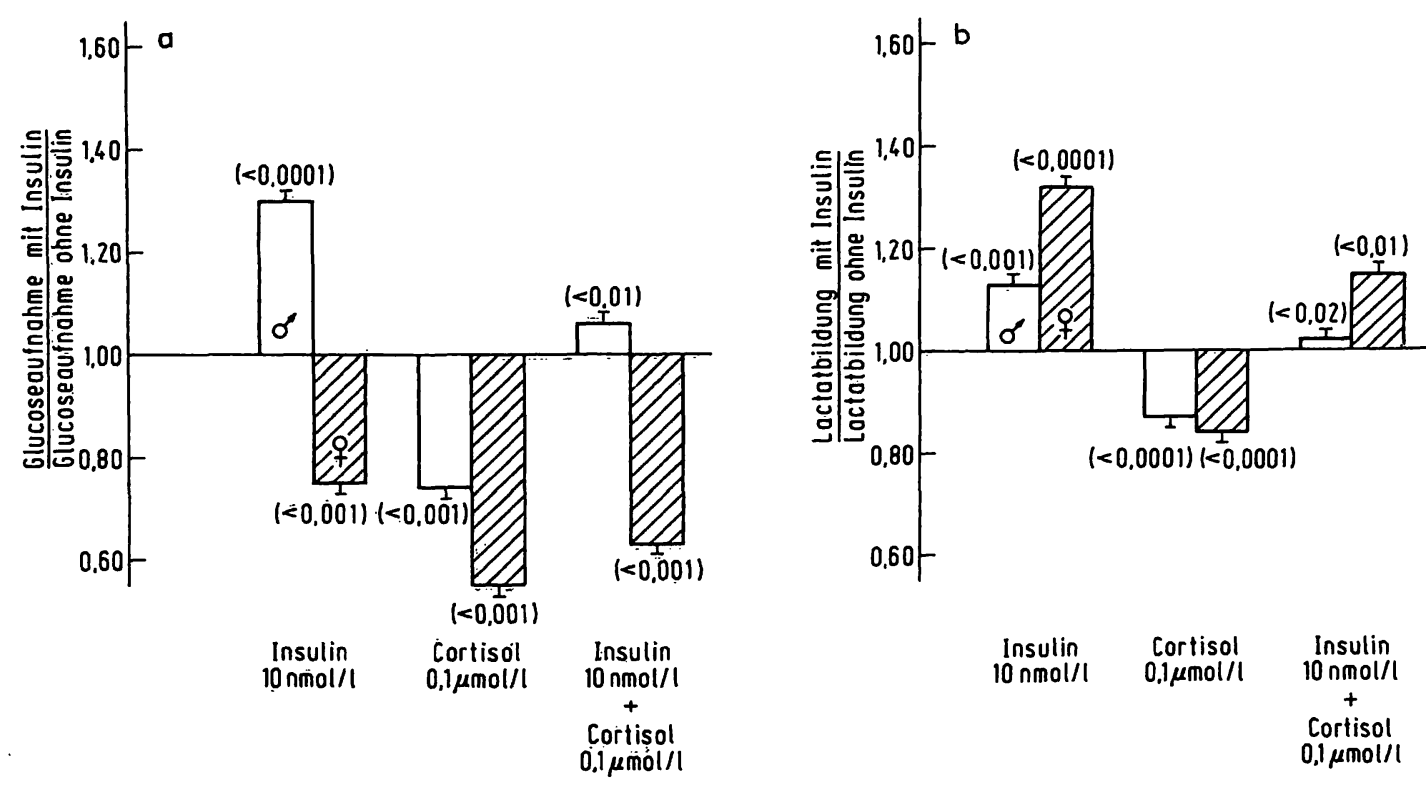

Abb. 3. Wirkung von Insulin und Cortisol auf die Glucoseaufnahme (a) und Lactatbildung (b) isolierter Thymocyten männlicher (nicht schraffierte Säulen) und weiblicher (schraffierte Säulen) Ratten (Gewicht: 140-150 g).

$40 \mathrm{mg}$ Zellen wurden in $0,9 \mathrm{ml}$ Krebs-Ringer-Bicarbonat-Puffer mit 5,5 mmol/1 Glucose und den angegebenen Hormonen $2 \mathrm{~h}$ bei $37^{\circ} \mathrm{C}$ inkubiert.

In den Kontrollansätzen (ohne Hormone) betrug die Glucoseaufnahme (a) auf Gramm Feuchtgewicht bezogen 18,89 1,23 $\mu \mathrm{mol} / 2 \mathrm{~h}\left(\delta^{\circ}\right)$ bziw. $24,99 \pm 1,34 \mu \mathrm{mol} / 2 \mathrm{~h}(9)$. Die Lactatbildung der Kontrollen betrug pro Gramm Feuchtgeivicht $6,34 \pm 0,37$ $\mu \mathrm{mol} / 2 \mathrm{~h}\left({ }^{\circ}\right)$ bzw. $5,45 \pm 0,41 \mu \mathrm{mol} / 2 \mathrm{~h}(\%)$.

Die Werte sind Mittelwerte $\left(\overline{\mathrm{x}} \pm s_{\overline{\mathrm{x}}}\right)$ aus 5 Präparationen. Die p-Werte für die Differenzen zwischen den Ansätzen mit und ohne Hormon (Kontrollen) sind in Klammern angegeben. 


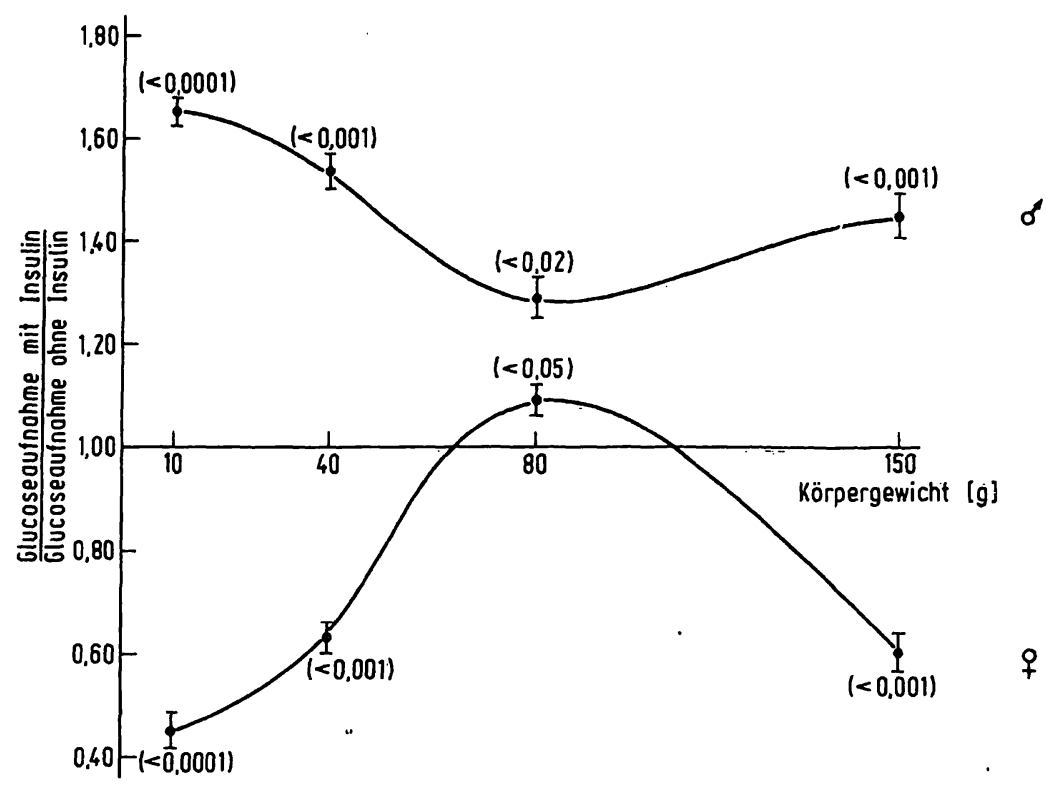

Abb. 4. Insulinwirkung auf die Glucoseaufnahme isolierter Thymocyten männlicher und weiblicher Ratten in Relation zum Körpergewicht.

$40 \mathrm{mg}$ Zellen wurden in $0,9 \mathrm{ml}$ Krebs-Ringer-Bicarbonat-Puffer mit 5,5 mmol Glucose und $10 \mathrm{nmol} / 1 \mathrm{Insulin} 2 \mathrm{~h}$ bei $37^{\circ} \mathrm{C}$ inkubiert.

In den Kontrollansätzen (ohne Hormon) betrug die Glucoseaufnahme berechnet in $\mu$ mol pro Gramm Feuchtgewicht und $2 \mathrm{~h}$ bei Thymocyten der Tiere mit einem Körpergewicht von $10 \mathrm{~g}: \delta^{\circ} 11,93, \odot 10,06 ; 40 \mathrm{~g}:$ o $15,33, \odot 14,71 ; 80 \mathrm{~g}:$ o 20,61 , ९ 23,58; $150 \mathrm{~g}:{ }^{\circ} 19,00$, \& 25,50.

Die Werte sind Mittelwerte $\left(\overline{\mathrm{x}} \pm s_{\bar{x}}\right)$ aus 5-8 Einżelpräparationen. Für jede Einzelpräparation wurden bei $10 \mathrm{bzw} .40 \mathrm{~g}$ schweren Ratten die Organe von 20 bzw. 10 Tieren verwendet. Bei 80 und $150 \mathrm{~g}$ schweren Tieren enthielt eine Einzelpräparation die Organe von jeweils 5 Ratten.

Die p-Werte für die Differenzen zwischen der Glucoseaufnahme der Inkubation mit und ohne Insulin (Kontrollen) sind in Klammern angegeben.

einer signifikanten Steigerung der Glucoseaufnahme. Die Insulinwirkungskurve zeigt ein Minimum bei $80 \mathrm{~g}$ schweren männlichen Ratten. Eine hemmende Wirkung von Insulin auf die Glucoseaufnahme durch Thymocyten weiblicher Ratten wurde bei Tieren mit einem Körpergewicht von 10,40 und $150 \mathrm{~g}$ registriert, während Thymocyten $80 \mathrm{~g}$ schwerer weiblicher Ratten auf Insulin mit einer geringen, aber signifikanten Erhöhung der Glucoseaufnahme reagierten. $\mathrm{Ob}$ die beiden Schnittpunkte der Insulinwirkungskurve weiblicher Ratten mit der Abszisse auf Körpergewichte schließen lassen, bei denen. die Thymocyten keinen Insulineffekt auf die Glucoseaufnahme erkennen lassen, ist noch nicht geklärt.

Um die Relevanz von Teilaspekten dieser Studie für lymphatische Gewebe des Menschen zu überprüfen, untersuchten wir die Insulinsensibilität isolierter Thymocyten nicht diabetischer Kinder. In Abbildung 5 ist der Effekt von $10 \mathrm{nmol} / 1$ Insulin auf die Glucoseaufnahme (Abb. $5 \mathrm{a}$ ) und Lactatfreisetzung (Abb. 5b) isolierter Thymocyten von Jungen und Mädchen dargestellt. Bei Thymocyten von Jungen stimulierte Insulin signifikant die Glucoseaufnahme und Lactatbildung. Die Zellen der Mädchen reagierten dagegen auf Insulin mit einer Verminderung der Glucoseaufnahme, während die Lactatfreisetzung erhöht wurde. Das Reaktionsmuster menschlicher Thymocyten zeigt, analog zu den lymphatischen Zellen der Ratte, eine hoch signifikante Geschlechtsspezifität.

\section{Diskussion}

Die Untersuchung zeigt die Insulinsensibilität lymphatischer Organe der Ratte und des Menschen. Die Hormonwirkung auf die Glucoseaufnahme und Lactatbildung der isolierten Thymus- und Milzzellen der Ratte konnte in fast allen Experimenten ab einer Insulinkonzentration von $0,1 \mathrm{nmol} / \mathrm{l}$ statistisch gesichert werden. Bei niedrigeren Insulinkonzentrationen wurden die Effekte deutlicher variabler und konnten nur noch teilweise statsitisch ábgesichert werden. Eine Interpretation der angedeuteten Hemmwirkung niedriger Insulinkonzentrationen ( $10 \mathrm{pmol} / \mathrm{l})$ auf die Glucoseaufnahme und Lactatbildung von Thymocyten und Milzzellen männlicher Tiere ist zur Zeit nicht möglich.

Goldfine et al. (14) fanden bei Untersuchungen der Insulinwirkung auf den $\alpha$-Aminoisobuttersäure-Einstrom in Rattenthymocyten gesicherte Hormoneffekte ab einer Insulinkonzentration .1. nmol/1. Unter der Vor- 

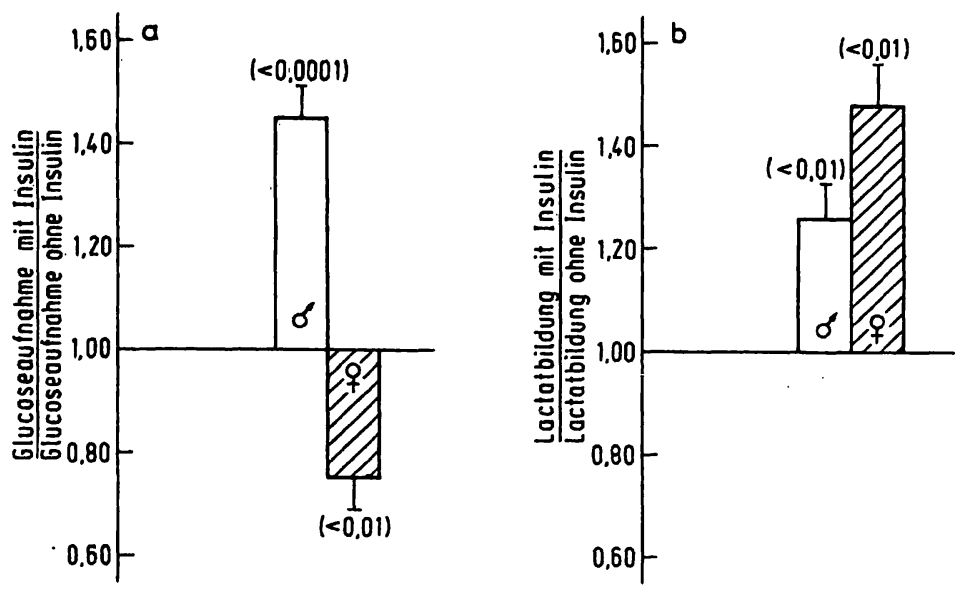

Abb. 5. Insulinwirkung auf die Glucoseaufnahme (a) und Lactatbildung (b) isolierter Thymocyten des Menschen (Alter: 2 Monate bis 10 Jahre).

$40 \mathrm{mg}$ Zellen wurden in $0,9 \mathrm{ml} \mathrm{Krebs-Ringer-Bicarbonatpuffer} \mathrm{mit} 1,1 \mathrm{mmol} / 1$ Glucose und $10 \mathrm{nmol} / 1 \mathrm{Insulin} 60 \mathrm{~min}$ bei $37^{\circ} \mathrm{C}$ inkubiert.

Glucoseaufnahme (a) in den Kontrollansätzen (ohne Insulin): o $2,83 \pm 0,86 \mu \mathrm{mol} / \mathrm{h} \cdot \mathrm{g}$ Feuchtgewicht, $84,37 \pm 0,86 \mu \mathrm{mol} / \mathrm{h} \cdot \mathrm{g}$ Feuchtgewicht. Lactatbildung (b) in den Kontrollansätzen: $\delta 5,62 \pm 0,64 \mu \mathrm{mol} / \mathrm{h} \cdot \mathrm{g}$ Feuchtgewicht, $83,02 \pm 1,01 \mu \mathrm{mol} / \mathrm{h} \cdot \mathrm{g}$ Feuchtgewicht.

Die Werte sind Mittelwerte $\left(\overline{\mathrm{x}} \pm \mathrm{s}_{\mathbf{x}}\right)$ aus 5-8 Präparationen. Die p-Werte für dic Differenzen zivischen den Ansätzen mit und ohne Insulin (Kontrollen) sind in Klammern angegeben.

aussetzung gleicher Qualität der Thymocytenpräparation reagierten Glucosetransport und Lactatbildung deutlich empfindlicher auf Insulin als die Aufnahme der $\alpha$-Aminoisobuttersäure. Die Insulinsensibilität der Thymocyten scheint geringer als die isolierter Fettzellen zu sein. Gammeltoft et al. (18) fanden in Fettzellen eine halbmaximale Stimulation der Lipogenese aus $\left[{ }^{3} \mathrm{H}\right]$ Glucose bei einer Insulinkonzentration von $70 \mathrm{pmol} / 1$ und eine maximale Stimulation bei Konzentrationen von weniger als $1 \mathrm{nmol} / 1$. Die Dosis-Wirkungskurve für den Insulineffekt auf die Glucoseoxidation isolierter Fettzellen hat eine ähnliche Charakteristik $(19,20)$. Die Ursachen der geringeren Hormonsensibilität isolierter Thymocyten könnten bereits bei der Bildung des Hormon-Rezeptorkomplexes zu suchen sein. Goldfine et al. (14) errechneten für den [ $\left.{ }^{125} \mathrm{~J}\right]$ Insulin-Rezeptorkomplex auf der Plasmamembran von Rattenthymocyten Dissoziationskonstanten der spezifischen Bindungsstellen hoher und niedriger Affinität in der Größ̣enordnung von $9 \mathrm{nmol} / 1$ bzw. $0,1 \mu \mathrm{mol} / 1$. Verglichen mit isolierten Fettzellen und Fettzellmembranen, bei denen mehrere Arbeitsgruppen $(18,19,21)$ nur eine Rezeptorklasse mit einer Dissoziationskonstanten von $50 \mathrm{pmol} / 1$ (19) bis maximal $7,1 \mathrm{nmol} / 1$ (21) gefunden haben, wäre die Affinität der Insulinrezeptoren isolierter Thymocyten niedriger als die der Fettzellen. Bei einem solchen Vergleich muß allerdings einschränkend festgestellt weerden, daß aufgrund verschiedener Techniken der Rezeptortitration und infolge des die Ińterpretation der Bindungṣdaten komplizierenden Vorliegens negativer Kooperation zwischen den Insulinrezeptoren (20) die in verschiedenen Laboratorien an ein und demselben Zelltyp bestimmten Dissoziationskonstanten für den Insulin-Rezeptorkomplex erheblich differieren können $(18,19,21)$. Die Untersuchungen von Gavin et al. (2), die an peripheren Lymphocyten des Menschen zwei Klassen von Insulin rezeptoren mit vergleichsweise niedrigen Dissoziationskonstanten für den Insulin-Rezeptorkomplex $\left(\mathrm{K}_{\mathrm{d} 1}=0,5 \mathrm{nmol} / 1, \mathrm{~K}_{\mathrm{d} 2}=7,1 \mathrm{nmol} / \mathrm{l}\right)$ nachgewiesen haben, könnten darauf hinweisen, daß die hohen Dissoziationskonstanten bei isolierten Thymuslymphocyten mit Vorbehalt zu bewerten sind. Interessanterweise ist die an frisch isolierten peripheren Lymphocyten (2) bestimmte Anzahl der Bindungsstellen (Rezeptorklasse 1:350 Bindungsstellen, Rezeptorklasse 2:1700 Bindungsstellen) im Vergleich zur Anzahl der Rezeptoren auf der Plasmamembran isolierter Fettzellen (11 000-50 000) $(18,19)$ um etwa eine Zehnerpotenz geringer. Wenn isolierte Thymuslymphocyten, wie vorläufige Untersuchungen unserer Arbeitsgruppe vermuten lassen, gleichfalls nur über eine niedrige $Z$ ahl von Insulinrezeptoren verfügen $\left(1 \times 10^{3}\right.$ Rezeptoren/Zelle), könnte auch darin einer der Gründe für eine verglichen mit Fettzellen geringere Insulinsensibilität zu finden sein. Nach cem Massenwirkungsgesetz ist bei der Reaktion:

$$
\text { Insulin }+ \text { Rezeptor } \rightleftharpoons \text { Insulin-Rezeptorkomplex }
$$

die Bildung des aktiven Insulinrezeptorkomplexes nicht nur von der Konzentration des freien Insulins, sondern ebenso entscheidend von der Konzentration der freien Hormonrezeptoren abhängig. Die ungewöhnliche $\mathrm{Ge}-$ schlechtsspezifität der Insulinwirkung auf die Glucoseaufnahme lymphatischer Zellen und die bei Ratten nachgewiesene Abhängigkeit des Insulineffektes vom Entwicklungsstadium der Ratte zeigen, wie stark die Reaktion auf Insulin durch endogene Faktoren moduliert wird. $\mathrm{Ob}$ es sich bei diesen Modulatoren um Hormone handelt, die 
die Lymphocyten direkt kontrollieren, läßt sich zur Zeit nicht sicher beurteilen, da lymphatische Organe einer vielfältigen hormonellen Steuerung unterliegen. Der Thymus steht zum Beispiel unter dem Einfluß von Glucocorticoiden, Östradiol, Testosteron, Thyroxin, Adrenalin, Somatotropin, Prolactin, Oxytocin, Vasopressin, Parathyrin und Calcitonin (23-27). Eine direkte Beteiligung von Östrogenen oder Androgenen an der Ausprägung der Geschlechtsspezifiiät der Insulinwirkung auf die Glucoseaufnahme schien nach den Experimenten an kastrierten Tieren zwar möglich, wurde aber anschlieBend durch die Untersuchungen am präpubertalen menschlichen Thymus nicht bestätigt. Diese Geschlechtsspezifität der Wirkung des Insulins auf die Glucoseaufnahme erklärt möglicherweise das von Goldfine et al. (14) berichtete Ausbleiben eines Insulineffektes auf den Influx von 3-O-Methylglucose in Rattenthymocyten. Goldfine et al. (14) führten nämlich die Versuche nicht gesondert an Thymocyten männlicher oder weiblicher Tiere durch, sondern an einem Pool von Thymocyten aus Ratten beiderlei Geschlechts. Der Nachweis, daß Insulin in dieser gemischten Zellpopulation eindeutig den Transport von $\alpha$-Aminoisobuttersäure (14) stimuliert, deutet auf das Fehlen einer vergleichbaren Geschlechtsspezifität der Insulinwirkung auf den Aminosäuretransport hin. Vorläufige Untersuichungen unserer Arbeitsgruppe (Bojar, H., Unnewehr, M. \& Staib, W., unveröffentlicht) zur Wirkung von Insulin auf die Aufnahme verschiedener Aminosäuren durch Rattenthymocyten scheinen diese Annahme zu bestätigen.

Zur weiteren Abklärung der Insulinwirkung auf die Glucoseutilisation isolierter Zellen lymphatischer Gewebe werden zur Zeit kinetische Untersuchungen der Hormonwirkung auf den Membrantransport radioaktiv markierter Glucosederivate durchgeführt.

\section{Danksagung}

Die Arbeit wurde durch Sachmittel der Deutschen Forschungsgemeinschaft im Rahmen des Sonderforschungṣbereichs 113 gefördert.

\section{Literatur}

1. Gavin, J. R., III, Roth, J., Jen, P. \& Freychet, P. (1972), Proc. Nat. Acad. Sci. USA 69, 747-751.

2. Gavin, J. R., III, Gordon, P., Roth, J., Archer, J. A. \& Buell, D. N. (1973), J. Biol. Chem. 248, 2202-2207.

3. Kahn, C. R., Freychet, P., Roth, J. \& Neville, D. M., Jr. (1974), J. Biol. Chem. 249, 2249-2257.

4. Freychet, P., Roth, J. \& Neville, D. M., Jr. (1971), Biochem. Biophys. Res. Commun. 43, 400-408.

5. Gammeltoft, S. \& Gliemann, J. (1973), Biochim. Biophys. Acta 320, 16-32.

6. Hadden, J. W., Hadden, E. M., Wilson, E. \& Good, R. A. (1972), Nature New Biol. 235, 174-177.

7. Martin, S. P., McKinney, G. R., Green, R. \& Becker, C. (1953), J. Clin. Invest. 32, 1171-1174.

8. Antonioli, J. A., Felber, J. P. \& Vannotti, A. (1967), Acta Haematol. 37, 161-173.

9. Kalant, N. \& Schucher, R. (1962), Canad. J. Biochem. 40, 899-903.

10. Mähr, G. (1971), Wien. Z. Inn. Med. 52, 19-26.

11. Krug, U., Krug, F. \& Cuatrecasas, P. (1972), Proc. Nat. Acad. Sci. USA 69, 2604-2608.

12. Helmreich, E. \& Eisen, H. N. (1959), J. Biol. Chem. 234, 1958-1965.

13. Boyett, J. D. \& Hofert, J. F. (1972), Horm. Metab. Res. 4, 163-167.

14. Goldfine, I. D., Gardner, J. D. \& Neville, D. M., Jr. (1972), J. Biol. Chem. 247, 6919-6926.
15. Werner, W., Rey, H. G. \& Wielinger, H. (1970), Z. Analyt. Chem. 252, 224-228.

16. Hohorst, H. J. (1962), in Methoden der enzymat. Analyse (H. U. Bergmeyer, Hrsg.), 1. Aufl., 266-270. Verlag Chemie, Weinheim/Bergstraße.

17. Matsui, N. \& Plager, J. E. (1969), Endocrinology 84, 1439-1449.

18. Gammeltoft, S. \& Gliemann, J. (1973), Biochim. Biophys. Acta 320, 16-32.

19. Cuatrecasas, P. (1971), Proc. Nat. Acad. Sci. USA 68, 1264-1268.

20. Freychet, P., Roth, J. \& Neville, D. M., Jr. (1971), Biochem. Biophys. Res. Commun. 43, 400-403.

21. Kono, T. \& Barham, F. W. (1971), J. Biol. Chem. 246, 6210-6216.

22. DeMeyts, P. (1973), Biochem. Biophys. Res. Commun. 55, 154-161.

23. Young, D. A. (1969), J. Biol. Chem. 244, 2210-2217.

24. Comsa, J. (1973), in Thymic Hormones (Luckey, T. D., ed.) $59-96$

25. MacManus, J. P., Whitfield, J. F. \& Youdale, T. (1971), J. Cell. Physiol. 77, 103-116.

26. Whitfield, J. F., Perris, A. D. \& Youdale, T. (1969), J. Cell. Physiol. 73, 203-212.

27. Whitfield, J. F., MacManus, J. P. \& Gillan, D. J. (1970), J. Cell. Physiol. 76, 65-76.

Dr. Hans Bojar

Institut für Physiologischen Chemie II der Universität Düsseldorf

Moorenstraße 5

D-4000 Düsseldorf 